\title{
An integrated injection seeded THz source and amplifier for time-domain spectroscopy
}

\author{
J. Maysonnave, ${ }^{1}$ N. Jukam, ${ }^{1 *}$ M. S. M. Ibrahim, ${ }^{2}$ K. Maussang, ${ }^{1}$ J. Madéo, ${ }^{1}$ P. Cavalié, ${ }^{1}$ P. Dean, ${ }^{2}$ S.P. \\ Khanna, ${ }^{2}$ D P. Steenson, ${ }^{2}$ E. H. Linfield, ${ }^{2}$ A. G. Davies, ${ }^{2}$ J. Tignon, ${ }^{1}$ S. S. Dhillon ${ }^{1}$ \\ ${ }^{1}$ Laboratoire Pierre Aigrain, Ecole Normale Supérieure, CNRS (UMR 8551), \\ Université P. et M. Curie, Université D. Diderot, 24 rue Lhomond 75231 Paris Cedex 05, France. \\ ${ }^{2}$ School of Electronic and Electrical Engineering, University of Leeds, Woodhouse Lane, Leeds LS9 2JT, U.K. \\ *Corresponding author: nathan.jukam@lpa.ens.fr
}

Received Month X, XXXX; revised Month X, XXXX; accepted Month X, XXXX; posted Month X, XXXX (Doc. ID XXXXX); published Month X, XXXX

\begin{abstract}
A terahertz (THz) frequency quantum cascade laser (QCL) is used as an integrated injection seeded source and amplifier for use in THz time-domain spectroscopy. A THz input pulse is generated inside a QCL, by illuminating the laser facet with a near-infrared pulse from a femtosecond laser, and amplified using gain switching. The THz output from the QCL is found to saturate upon increasing the amplitude of the THz input power, which indicates that the QCL is operating in an injection seeded regime. (C) 2011 Optical Society of America OCIS Codes: 300.6270, 300.6495, 140.5965
\end{abstract}

In the technique of terahertz (THz) frequency timedomain spectroscopy (TDS), $\mathrm{THz}$ radiation is generated and detected using femtosecond ( $\mathrm{fs}$ ) near-infrared laser pulses, enabling measurement of both the $\mathrm{THz}$ amplitude and phase [1]. In TDS systems, the THz radiation can be generated when the near-infrared fs laser illuminates a GaAs photo-conductive antenna [2], travels through an electro-optic crystal with a non-vanishing $\chi^{2}$ [3], or is focused in air [4]. In all these cases, the $\mathrm{THz}$ field amplitude scales linearly with the power of the fs pulse, until saturation takes place. One strategy to increase further $\mathrm{THz}$ field amplitudes is to use higher fs pulse energies, and TDS systems that utilize $\mathrm{mJ}$ pulse energies are becoming increasingly common [5]. Although this is a promising laboratory-based technique, it is not practical for applications outside the laboratory owing to the large cost and size of regenerative amplified laser systems.

An alternative approach to generate higher $\mathrm{THz}$ fields is to amplify $\mathrm{THz}$ waves directly after generation, and hence enable low-power fs laser pulses to generate high $\mathrm{THz}$ wave amplitudes. $\mathrm{THz}$ frequency quantum cascade lasers (QCLs) are natural candidates for $\mathrm{THz}$ amplifiers, and have already been used as external $\mathrm{THz}$ amplifiers to measure the QCL gain. [6-8]. In the steady-state, external amplification is limited by gain clamping and depends on the facet reflectivity [9]. Gain switching with RF pulses can be used to unclamp the gain in the QCL and augment external pulse amplification [10]. Amplifying THz 'seed' pulses in QCLs with sufficiently long RF pulses [11] can lead to coherent injection seeding where the QCL emission is phase-locked to the fs laser. This enables phase-resolved detection of the QCL emission in the timedomain, and permits the use of QCLs as intense $\mathrm{THz}$ sources for TDS. However, if the $\mathrm{THz}$ seed pulses are generated by external photoconductive antennas, the $\mathrm{THz}$ must be coupled from free space into the QCL cavity. This introduces large coupling losses that are associated impedance mismatch between the cavity and free space and can negate the effect of $\mathrm{THz}$ amplification.

In this letter, injection seeding of a QCL is achieved with $\mathrm{THz}$ seed pulses that are generated directly inside the
QCL cavity. This results in a completely integrated QCLbased source for TDS. Specifically the THz seed pulses are generated inside the QCL cavity [12] by illuminating one of the facets with a near-infrared fs laser beam (Fig 1a). This removes the need to use a photoconductive $\mathrm{THz}$ emitter to generate the $\mathrm{THz}$ seed pulses and parabolic mirrors to couple the $\mathrm{THz}$ seed pulses into the cavity (Fig 1b). Gain switching of the QCL is used to achieve large amplification of the internally generated $\mathrm{THz}$ seed pulses. A coupled cavity scheme allows the amplitude of the input seed pulse to be increased without affecting the QCL gain $[13,14]$. We find that for sufficient $\mathrm{THz}$ seed amplitudes, the amplified seed pulses saturate the QCL gain, which indicates that injection seeding takes place via integrated seed generation.

To enter the injection seeding regime, the amplitude of the $\mathrm{THz}$ pulse generated at the facet must be sufficiently large to prevent the QCL from being 'seeded' by amplified spontaneous emission. Since the $\mathrm{THz}$ pulse amplitude generated at the facet increases with applied voltage [12], it is therefore desirable to apply a large voltage across the facet. However, QCL operation ceases once the applied bias becomes too large owing to misalignment of the injector and upper level laser state. In order to decouple the facet voltage from the QCL bias voltage, focused ion beam (FIB) etching was used to remove a $20 \mu \mathrm{m}$ wide portion of the gold contact and the heavily-doped n-GaAs contact layer on the top of the laser ridge. This splits the QCL cavity into a short $305 \mu \mathrm{m}$ seeding section used as the $\mathrm{THz}$ pulse generator, and a long $2.5 \mathrm{~mm} \mathrm{QCL}$ gain section for $\mathrm{THz}$ pulse amplification. The resistance between these two sections $(\sim 35 \Omega)$ is several times larger than the QCL differential resistance (3-6 $\Omega$ ). Although, the two sections are not completely electrically isolated; removing the top contact in this way eliminates the need to etch through the entire active region using reactive ion etching [13,14]. As shown in Fig. 1d, independently biasing the seeding section decreases the QCL output power. This is most likely due to the leakage current between the seeding and QCL section and increased $\mathrm{THz}$ losses in the seeding section. The QCL is based on a 


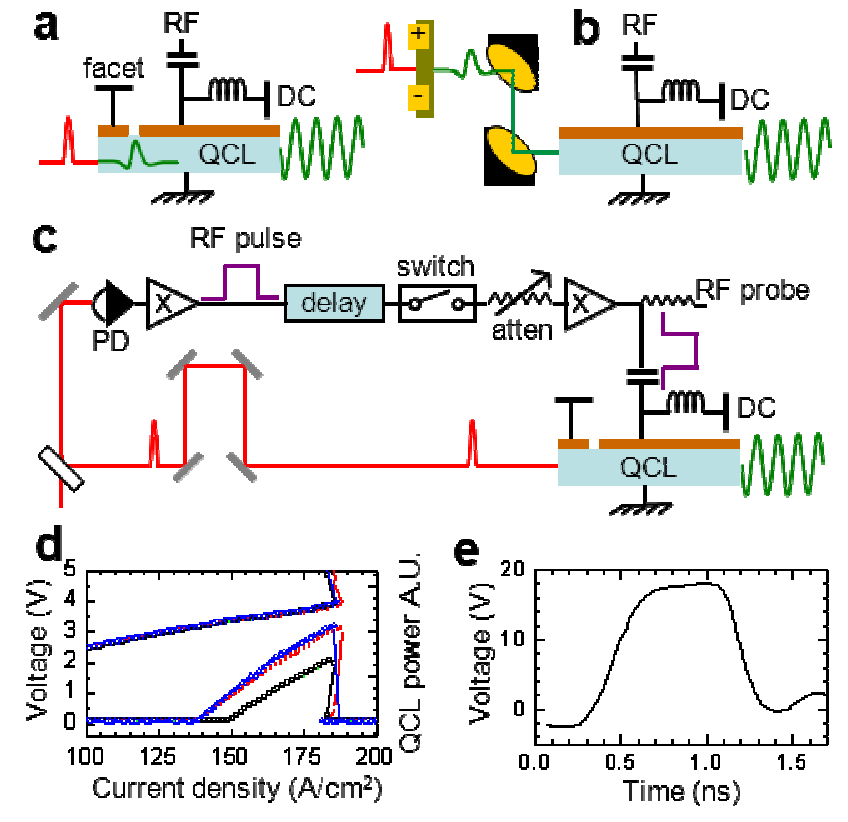

Figure 1 a) $\mathrm{THz}$ pulses are generated inside the QCL by illuminating the seeding section with an fs laser. b) $\mathrm{THz}$ pulses created by an external photoconductive antenna are coupled into a QCL with parabolic mirrors. c) RF pulses are created with a fast photodiode (PD), and amplified. An electronic delay line is used to vary the RF pulse arrival time. The RF pulse train is modulated with a $50 \Omega$ switch, and the pulse amplitude can be varied with a variable attenuator. A bias tee allows a quasi-DC voltage to be applied to the QCL along with the RF. A $20 \mathrm{~dB}$ pickoff tee (RF probe) allows the RF shape to be monitored. $d$ ) Lightcurrent density (V-J) plots for the device with the seeding section biased at $0 \mathrm{~V}$ (black), 3.8V $\left(\sim 180 \mathrm{~A} / \mathrm{cm}^{2}\right)$ (red), and shorted to the QCL section (blue). e) The RF pulse sent to the QCL measured

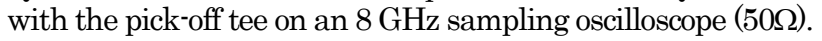

bound-to-continuum design, and processed into a single plasmon waveguide with a lateral dimension of $180 \mu \mathrm{m}$ and a total cavity length of $2.85 \mathrm{~mm}$. The QCL is soldered to a gold-plated copper mount and placed in a continuous flow cryostat. Measurements are taken at $10 \mathrm{~K}$, and laser emission is centered at $2.5 \mathrm{THz}$.

Before the arrival of the fs pulse (80fs, $808 \mathrm{~nm}, 6 \mathrm{~nJ})$ that generates a $\mathrm{THz}$ pulse in the seeding section, the gain section is biased below threshold. When the $\mathrm{THz}$ pulse is generated in the QCL, an RF bias pulse (triggered by the fs laser) is applied to the gain section. This places the QCL in a transient state where the gain is much greater than the clamped gain, and permits large amplification [10]. Fig. 1c shows a schematic diagram of the electronic arrangement. A fast photodiode generates the RF pulses when illuminated with the fs laser. The RF pulses are amplified by a pre-amplifier that is operated in the saturation regime to produce $\mathrm{RF}$ pulses with a relatively flat top. The RF pulses are subsequently amplified by a power amplifier and applied to the QCL. The power of the incident RF pulses (Fig. 1e) is $\sim 6 \mathrm{~W}$, and about $40 \%$ of the RF power is injected into the QCL. Faster electronics permit shorter $\mathrm{RF}$ rise times and pulse durations compared with studies of external injection seeding [11]. A bias tee also allows the QCL gain section to be biased with a quasi-DC voltage $(10 \mathrm{kHz})$, which reduces the $\mathrm{RF}$ power necessary to drive the QCL above threshold. The quasi$\mathrm{DC}$ bias is set to ensure that the RF pulses drive the QCL to the bias at which maximum gain occurs (i.e. just before misalignment of the QCL band-structure). The seeding section is biased at $3.8 \mathrm{~V}\left(\sim 180 \mathrm{~A} / \mathrm{cm}^{2}\right)$ which roughly corresponds with $\mathrm{J}_{\max }$ in Fig. 1d. The RF pulses and QCL gain section are modulated at $10 \mathrm{kHz}(25 \%$ duty cycle) while the seeding section is modulated at $20 \mathrm{kHz}$ i.e. at twice the duty cycle of the QCL gain section and RF pulses modulation. This permits lock-in detection of the difference field, which removes the unamplified low frequency components of the $\mathrm{THz}$ signal.

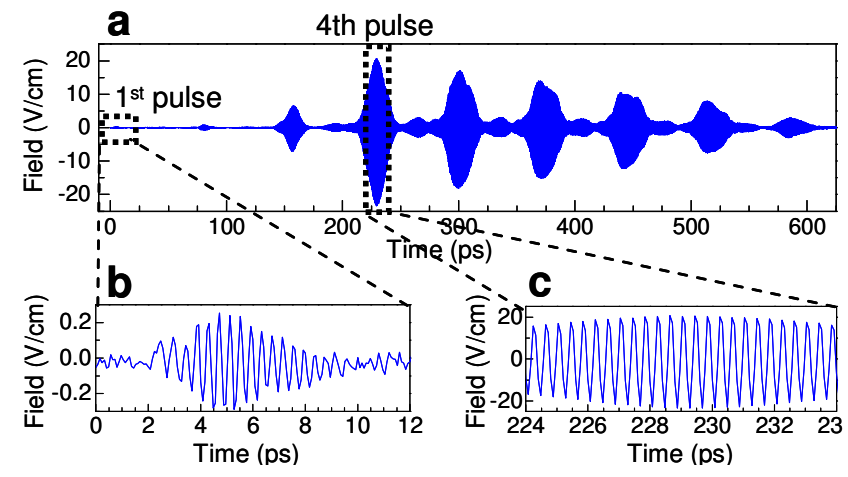

Figure 2 a) The $\mathrm{THz}$ difference field emitted from the QCL measured with electro-optic sampling. The incident RF pulse amplitude is $6 \mathrm{~W}$ and the quasi-DC bias is $62 \mathrm{~A} / \mathrm{cm}^{2}$. b) Enlarged view of the 1st $\mathrm{THz}$ pulse that undergoes a single pass through the QCL. c) Enlarged view of the 4th THz pulse, which shows the phase-resolved nature of the measurement.

Fig. 2 shows the QCL output when the RF drives the QCL above threshold. After the initial $\mathrm{THz}$ pulse is generated in the seeding section, it traverses the laser cavity and experiences gain (near the laser frequency) and losses (at other frequencies). At the opposite facet, part of the $\mathrm{THz}$ pulse is reflected back into the cavity and part is transmitted out of the QCL. This process will then continue with multiple passes traveling through the cavity. The output of the QCL thus consists of several $\mathrm{THz}$ pulses separated by the round-trip time ( $71 \mathrm{ps})$ of the cavity. A pair of parabolic mirrors collimates and focuses the transmitted $\mathrm{THz}$ pulses onto a $200-\mu \mathrm{m}$-thick $<110>$ $\mathrm{ZnTe}$ crystal for electro-optic detection.

As shown in Fig. 2a, the $\mathrm{THz}$ pulses undergo large amplification when the RF pulse brings the QCL bias above threshold and unclamps the gain. Figs. $2 \mathrm{~b}$ and $2 \mathrm{c}$ are zoomed in portions of the 1st and 4 th $\mathrm{THz}$ pulses in Fig $2 \mathrm{a}$, that show the phase-resolved nature of the measurement in Fig. 2a. After three round-trips the difference field is amplified by more than a factor of 80 , and the output field is almost two orders of magnitude larger than the input field. The amplified $\mathrm{THz}$ emission lasts for approximately the same time as the $R F$ pulse duration in Fig. 1e. Since there is no significant reflection between the seeding section and the gain section, no secondary $\mathrm{THz}$ seed pulses are produced. Although later $\mathrm{THz}$ pulses undergo dispersion, the $\mathrm{THz}$ pulses remain 
distinct. The maximum peak-to-peak field $(40 \mathrm{~V} / \mathrm{cm}$ corresponding to an intensity of $1.8 \mathrm{~W} / \mathrm{cm}^{2}$ ) is significantly greater than studies of external injection seeding on a $2.1 \mathrm{THz}$ QCL [11], and is due to the higher output powers of the $2.5 \mathrm{THz}$ devices $(\sim 20 \mathrm{~mW})$ compared to the $2.1 \mathrm{THz}$ device $(1.5 \mathrm{~mW})$. From the ratio of the spectral amplitudes of successive $\mathrm{THz}$ pulses in Fig. 2a, we can find the average difference between the unclamped gain and the clamped gain, which we define as the excess gain. A positive excess gain will result in a net amplification of a pulse that undergoes a round-trip through the QCL cavity. The largest value of the average excess gain (at the laser frequency) is $9 \mathrm{~cm}^{-1}$ and occurs between the $2^{\text {nd }}$ and $3^{\text {rd }}$ pulses in Fig. 2a.

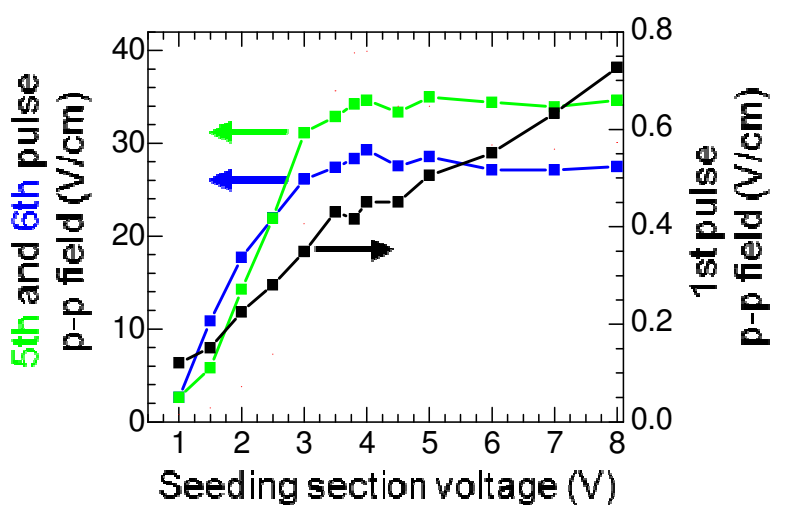

Figure 3 Maximum peak-to-peak ( $\mathrm{p}-\mathrm{p}$ ) fields of the 1st (black), fifth (green) and sixth (blue) $\mathrm{THz}$ pulses versus the voltage applied to the seeding facet. For values of the seeding voltage $>4$ $\mathrm{V}$, the amplitudes of the fifth and sixth $\mathrm{THz}$ pulses saturate, while the amplitude of the 1st $\mathrm{THz}$ pulse continues to increase.

To determine if the $\mathrm{THz}$ input pulses saturate the QCL gain (and are thus injection seeding the QCL), the output QCL field can be studied as function of the input $\mathrm{THz}$ pulse amplitude by varying the voltage applied to the seeding section. The peak-to-peak fields of the 1st, 5th and 6th output THz pulses are plotted in Fig. 3 as a function of the seeding section voltage. The 1st output $\mathrm{THz}$ pulse corresponds to a single pass through the cavity. Although the $\mathrm{THz}$ radiation generated at the facet cannot be directly measured, it is proportional to the amplitude of the 1st emitted $\mathrm{THz}$ pulse. The amplitude of this pulse increases almost linearly for seeding section voltage less than 3.5 V. For higher voltages, the input amplitude continues to increase with seeding section voltage although the slope decreases slightly. When the seeding section voltage (and hence the amplitude of the generated $\mathrm{THz}$ pulse) is small, the peak-to-peak field of the 5th and 6 th pulses also increases with voltage. However, for large seeding voltages the peak amplitudes of the 5th and 6th pulses are flat even though the amplitude of the 1st pulse keeps increasing. This indicates that the gain of the laser is saturated (clamped) by the amplified $\mathrm{THz}$ pulses, and the laser emission has entered the injection seeded regime.
In conclusion, we have demonstrated an integrated QCL-based source of $\mathrm{THz}$ radiation suitable for TDS. $\mathrm{THz}$ pulses are generated directly inside a coupled-cavity QCL by illuminating the facet with fs near-infrared laser pulses, and are subsequently amplified to high field values using gain switching until injection seeding of the QCL is achieved. The THz signal generated in the seed element is thus increased by amplifying the $\mathrm{THz}$ wave using the high gain available from the QCL gain section, rather than by increasing the fs laser power. Decoupling the strength of the $\mathrm{THz}$ signal from the fs laser power could make TDS more practical by reducing the need for higher power fs lasers.

Acknowledgements This work was supported by ANR projects "HI-TEQ", "THINQE-PINQE", ERC Advanced Grant projects "NOTES" and "TOSCA", the EPSRC (UK), and the MARA Agency (Malaysia).

\section{References}

1. M. Hangyo, M. Tani, and T. Nagashima, Int. J. of Inf. Mill. Waves. 26, 1661 (2005).

2. Dreyhaupt, S. Winnerl, T. Dekorsy, and M. Helm, Appl. Phys. Lett. 86, 121114 (2005).

3. T. Löffler, T. Hahn, M. Thomson, F. Jacob, and H. Roskos, Opt. Express 13, 5353 (2005).

4. T. Bartel, P. Gaal, K. Reimann, M. Woerner, and T. Elsaesser Opt. Lett. 30, 2805 (2005).

5. M. C. Hoffman, and J. A. Fulop, J. Phys. D. App. Phys. 44, 03001 (2011).

6. J. Kröll, J. Darmo, S. S. Dhillon X. Marcadet, M. Calligaro, C. Sirtori, and K. Unterrainer, Nature 449, 698 (2007).

7. N. Jukam, S. S. Dhillon, D. Oustinov, Z.-Y. Zhao, S. Hameau, J. Tignon, S. Barbieri, A. Vasanelli, P. Filloux, C. Sirtori, and X. Marcadet, Appl. Phys. Lett. 93, 101115 (2008).

8. N. Jukam, S. S. Dhillon, D. Oustinov, J. Madéo, J. Tignon, R. Colombelli, P. Dean, M. Salih, S. P. Khanna, E. H. Linfield, and A. G. Davies, Appl. Phys. Lett. 94, 251108 (2009).

9. R. Rungsawang, N. Jukam, J. Maysonnave, P. Cavalie, J. Madeo, D. Oustinov, S.S. Dhillon, J. Tignon, P. Gellie, C. Sirtori, S. Barbieri, H.E. Beere, D.A. Ritchie Appl. Phys. Lett. 98, 101102 (2011).

10. Nathan Jukam, Sukhdeep S. Dhillon, D. Oustinov, J. Madeo, C. Manquest, S. Barbieri, Carlo Sirtori, Suraj P. Khanna, E. H. Linfield, A. G. Davies, and J. Tignon, Nat. Phot. 3, 715 (2009).

11. D. Oustinov, N. Jukam, R. Rungsawang, J. Madéo, Stefano Barbieri, P Filloux, C. Sirtori, X. Marcadet, J. Tignon, and S. Dhillon, Nat. Comm. 1, 69 (2010).

12. S. S. Dhillon, S. Sawallich, N. Jukam, D. Oustinov, J. Madéo, S. Barbieri, P. Filloux, C. Sirtori, X. Marcadet and J. Tignon, Appl. Phys Lett. 96, 061107 (2010).

13. M. Martl, J. Darmo, C. Deutsch, M. Brandstetter, A. M. Andrews, P. Klang, G. Strasser, and K Unterrainer, Optics Express 19, 733 (2011).

14. D. Burghoff, T-Yu Kao, D. Ban, A. Lee, M. Wie, Q. Hu, and J. Reno, Appl. Phys. Lett. 98, 061112 (2011). 


\section{References}

1) M. Hangyo, M. Tani and T. Nagashima, "Terahertz Time-Domain Spectroscopy of Solids: A Review" International Journal of Infrared and Millimeter Waves 26, (12) 1661-1690, (2005).

2) Dreyhaupt, S. Winnerl, T. Dekorsy, and M. Helm, "High-intensity terahertz radiation from a microstructured largearea photoconductor", Appl. Phys. Lett. 86, 121114-121114-3 (2005).

3) T. Löffler, T. Hahn, M. Thomson, F. Jacob, and H. Roskos, "Large-area electro-optic ZnTe terahertz emitters", Optics Express 13, (14) 5353-5362 (2005).

4) T. Bartel, P. Gaal, K. Reimann, M. Woerner, and T. Elsaesser, "Generation of single-cycle THz transients with high electric-field amplitudes”, Optics Letters, 30 (20) 2805-2807 (2005).

5) M. C Hoffmann and J. A. Fülöp, "Intense ultrashort terahertz pulses: generation and applications", Journal of Physics D: Applied Physics 44, (8) 083001 (2011).

6) J. Kröll, J. Darmo, S. S. Dhillon X. Marcadet, M. Calligaro, C. Sirtori and K. Unterrainer "Phase-resolved measurements of stimulated emission in a laser", Nature 449, 698-701 (2007).

7) N. Jukam, S. S. Dhillon, D. Oustinov, Z.-Y. Zhao, S. Hameau, J. Tignon, S. Barbieri, A. Vasanelli, P. Filloux, C. Sirtori, and X. Marcadet, "Investigation of spectral gain narrowing in quantum cascade lasers using terahertz time domain spectroscopy”, Appl. Phys. Lett. 93, 101115-101115-3 (2008).

8) N. Jukam, S. S. Dhillon, D. Oustinov, J. Madéo, J. Tignon, R. Colombelli, P. Dean, M. Salih, S. P. Khanna, E. H. Linfield, and A. G. Davies, "Terahertz time domain spectroscopy of phonon-depopulation based quantum cascade lasers", Appl. Phys. Lett. 94, 251108 (2009).

9) R. Rungsawang, N. Jukam, J. Maysonnave, P. Cavalie, J. Madeo, D. Oustinov, S.S. Dhillon, J. Tignon, P. Gellie, C. Sirtori, S. Barbieri, H.E. Beere, D.A. Ritchie, "Gain enhancement in a terahertz quantum cascade laser with parylene antireflection coatings", Appl. Phys. Lett. 98, (10) 101102-101102-3 (2011).

10) N. Jukam, S. S. Dhillon, D. Oustinov, J. Madeo, C. Manquest, S. Barbieri, Carlo Sirtori, Suraj P. Khanna, E. H. Linfield, A. G. Davies and Jérôme Tignon, "Terahertz amplifier based on gain switching in a quantum cascade laser", Nature Photonics 3, 715 - 719 (2009).

11) D. Oustinov, N. Jukam, R. Rungsawang, J. Madéo, Stefano Barbieri, P Filloux, C. Sirtori, X. Marcadet, J. Tignon S. Dhillon "Phase seeding of a terahertz quantum cascade laser", Nature Communications 1, 69 (2010).

12) S. S. Dhillon, S. Sawallich, N. Jukam, D. Oustinov, J. Madéo, S. Barbieri, P. Filloux, C. Sirtori, X. Marcadet, and J. Tignon, "Integrated terahertz pulse generation and amplification in quantum cascade lasers", Appl. Phys. Lett. 96, 061107-061107-3 (2010).

13) M. Martl, J. Darmo, C. Deutsch, M. Brandstetter, A. M. Andrews, P. Klang, G. Strasser, and K Unterrainer, "Gain and losses in THz quantum cascade laser with metal-metal waveguide”, Optics Express 19, (2) 733-738 (2011).

14) D. Burghoff, T-Yu Kao, D. Ban, A. Lee, M. W. Q. Hu, J. Reno, "A terahertz pulse emitter monolithically integrated with a quantum cascade laser", Appl. Phys. Lett. 98, (6) 061112 - 061112-3 (2011). 